\title{
EXTERIOR INTERFACE CRACKS
}

\author{
MARIA COMNINOU \\ Department of Civil Engineering, University of Michigan, Ann Arbor, MI 48109, U.S.A. \\ U.S.A. \\ (Communicated by I. N. SNEDDON)
}

\begin{abstract}
Continuing a recent investigation of interface cracks, attention is paid to the exterior crack. Two elastic solids bonded over a finite segment of their boundary and capable of transmitting shear and tensile tractions are considered. It is found that one of the edge cracks remains completely closed under shear alone, and opens gradually as the level of tension is increased. Both crack tips, however, must remain closed at least over a small interval. Stress intensity factors and bond and contact stresses are given for a specific example.
\end{abstract}

\section{INTRODUCTION}

RECENT WORK by the author $[1,2]$ has shown how to avoid contradictions previously present in the conventional solution of interface crack problems [3]. The new formulation allows the crack to remain partially closed near the tips, so that neither overlapping in the zone of separation nor tensile tractions in the contact zones occur. A few of the basic problems in this context have been considered already: the interface crack in a combined tension-compression and shear field [4], the penny-shaped crack at an interface [5] and the periodic array of interface cracks [6]. Some interesting and unexpected results were discovered: if the interface crack extends along the interface and does not veer into one of the solids, the propagation is according to mode II (shear) only, regardless of the type of loading. If the external load is shear, no amount of uniform compression can completely close the crack, which then resembles a bubble filled with fluid [4]. The 'bubble' phenomenon was recently observed also by Janach [7] who did a finite element analysis of an edge crack between an elastic-rigid interface subjected to a normal compression and a lateral compression simulating the effect of shear. The correction required to remove the objectionable singularity at the crack tip was not incorporated by Janach, and mode I stress intensity factors were computed.

In the present article we consider the problem of two dissimilar half spaces bonded along their boundary over a segment of length $2 L$ as shown in Fig. 1 . The common boundary (viz. the bond and the contact zones that develop) transmits known normal and shear stress resultants due to applied loads at infinity. This problem had been solved originally by Erdogan [8], who however, disregarded the overlapping of the crack faces. The new analysis predicts that one of the edge cracks remains completely closed if no tension is transmitted by the bond, and thus significantly departs from the former solution.

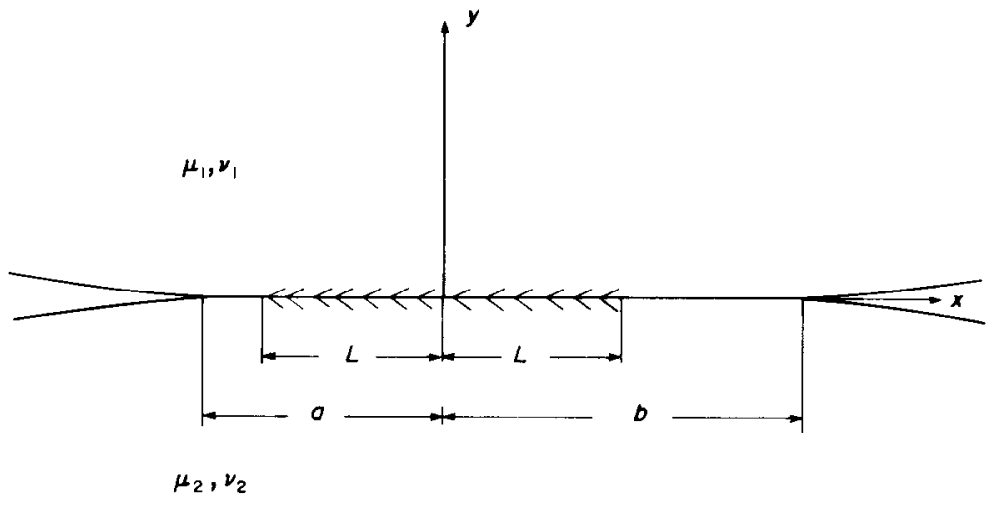

Fig. 1. Exterior interface crack. 


\section{FORMULATION}

Consider two elastic solids with shear moduli $\mu_{1}$ and $\mu_{2}$ and Poisson's ratios $\nu_{1}$ and $\nu_{2}$. The solids are perfectly bonded over a segment of length $2 L$ as shown in Fig. 1. Due to the action of external loads, the common boundary transmits normal tractions with the resultant $P$ and shear tractions with the resultant $Q$, where $P$ and $Q$ are taken as positive constants. The conventional analysis, which assumes both edge cracks completely open, leads to oscillatory singularities at the crack tips and predicts material interpenetration of finite extent. For instance, if $P$ is zero, overlapping occurs over the entire length of the right or left edge crack, depending on the material combination. Thus, the two cracks must be partially closed over the zones $(-a,-L)$ and $(L, b)$ where $a$ and $b$ are unknown constants. The contact is assumed frictionless.

To formulate the problem advantage is taken of the Flamant solution [9]. The quantities of interest here are the slopes of the displacements along the $x$-axis at the interface. To obtain these from the Flamant solution the limits must be taken with some care. If use is made of the identities [10]

$$
\begin{array}{cc}
\lim _{y \rightarrow 0} \frac{y}{x^{2}+y^{2}}=\pi \delta(x), & y>0 \\
\lim _{y \rightarrow 0} \frac{y^{3}}{\left(x^{2}+y^{2}\right)^{2}}=\frac{\pi}{2} \delta(x), & y>0
\end{array}
$$

the results for a concentrated force $\left(F_{x}, 0\right)$ at $x=\xi, y=0$ acting on the lower half space are

$$
\begin{gathered}
\left.\frac{\partial u_{x}^{(2)}}{\partial x}\right|_{y=0}=-\frac{F_{x}\left(\kappa_{2}+1\right)}{4 \mu_{2} \pi} \frac{1}{x-\xi} \\
\left.\frac{\partial u_{y}^{(2)}}{\partial x}\right|_{y=0}=-\frac{F_{x}\left(\kappa_{2}-1\right)}{4 \mu_{2}} \delta(x-\xi),
\end{gathered}
$$

and for a force $\left(0, F_{y}\right)$

$$
\begin{aligned}
& \left.\frac{\partial u_{x}^{(2)}}{\partial x}\right|_{y=0}=-\frac{F_{y}\left(\kappa_{2}-1\right)}{4 \mu_{2}} \delta(x-\xi) \\
& \left.\frac{\partial u_{y}^{(2)}}{\partial x}\right|_{y=0}=-\frac{F_{y}\left(\kappa_{2}+1\right)}{4 \mu_{2} \pi} \frac{1}{x-\xi} .
\end{aligned}
$$

In these expressions $\delta(x)$ denotes the Dirac delta function and $\kappa=3-4 \nu$ for plane strain.

The normal traction considered as a distribution of normal forces along the common boundary $(-a, b)$ is denoted by $p(x)$ and the shear traction being a distribution of tangential forces over the bond $(-L, L)$ by $q(x)$. In this formulation several boundary conditions are automatically satisfied: continuity of tractions over the common boundary of the solids $(-a, b)$, vanishing shear tractions over the contact zones $(-a,-L),(L, b)$ and vanishing tractions over the separation zones $x<-a, x>b$. The remaining boundary conditions are continuity of normal displacements over $(-a, b)$ and continuity of tangential displacements over $(-L, L)$. Instead of matching the displacements, however, it is more convenient to match the slopes at the interface, since a constant translation will not affect the solution. Thus, with no loss of generality

$$
\begin{array}{cc}
\frac{\partial u_{x}^{(1)}}{\partial x}=\frac{\partial u_{x}^{(2)}}{\partial x}, & -L<x<L \\
\frac{\partial u_{y}^{(1)}}{\partial x}=\frac{\partial u_{y}^{(2)}}{\partial x}, & -a<x<b .
\end{array}
$$

Using (3)-(6), the boundary conditions (7) and (8) for the distributions $p(x)$ and $q(x)$ become

$$
\beta p(x)-\frac{1}{\pi} \int_{-L}^{L} \frac{q(\xi)}{\xi-x} \mathrm{~d} \xi=0, \quad-L<x<L
$$




$$
\beta q(x)+\frac{1}{\pi} \int_{-a}^{b} \frac{p(\xi)}{\xi-x} \mathrm{~d} \xi=0, \quad-a<x<b
$$

where

$$
\beta=\frac{\mu_{2}(\kappa-1)-\mu_{1}\left(\kappa_{2}-1\right)}{\mu_{2}\left(\kappa_{1}+1\right)+\mu_{1}\left(\kappa_{2}+1\right)}, \quad-\frac{1}{2}<\beta<\frac{1}{2} .
$$

The conditions of the stress resultants are

$$
\begin{aligned}
& \int_{-L}^{L} q(\xi) \mathrm{d} \xi=Q \\
& \int_{-a}^{b} p(\xi) \mathrm{d} \xi=P .
\end{aligned}
$$

Equations (9) and (10) are a system of coupled singular integral equations with Cauchy kernels that must be solved in conjunction with the constraints (12) and (13). The function $q(x)$ is square-root singular at $L$ and $-L[1]$ and $p(x)$ is bounded at $-a$ and $b$. However, if the parameter $\beta$ is taken as positive, the left zone is expected to be extremely small and $p(x)$ which is singular at $-L$ and $L$ outside the bond changes very rapidly. In such case it is computationally better to take $p(x)$ singular at $-a$ and then impose the condition that the intensity of the singularity vanishes there. Under this assumption (10) is solved formally [11] for $p(x)$

$$
p(x)=\frac{\beta}{\pi} w(x) \int_{-L}^{L} \frac{q(\xi)}{w(\xi)(\xi-x)} \mathrm{d} \xi
$$

where

$$
w(x)=(b-x)^{1 / 2}(x+a)^{-1 / 2} .
$$

Using (14), eqn (13) becomes

$$
\int_{-L}^{L} \frac{q(\xi)}{w(\xi)} \mathrm{d} \xi=\frac{P}{\beta}
$$

Substituting (14) into (9) we obtain

$$
\beta^{2} w(x) \int_{-L}^{L} \frac{q(\xi)}{w(\xi)(\xi-x)} \mathrm{d} \xi-\int_{-L}^{L} \frac{q(\xi)}{\xi-x} \mathrm{~d} \xi=0 .
$$

\section{NUMERICAL SOLUTION AND RESULTS}

Equation (17) is a Cauchy singular integral equation for $q(x)$ and must be solved numerically. Using the dimensionless variables

$$
s=\frac{x}{L}, \quad r=\frac{\xi}{L}
$$

retaining the same symbols for the new functions and setting

$$
q(s)=\frac{Q}{L}\left(1-r^{2}\right)^{-1 / 2} \phi(r)
$$

(17), (12), (16) and (14) become

$$
\int_{-1}^{1} \frac{\left(1-r^{2}\right)^{-1 / 2}}{r-s} \phi(r)\left[\beta^{2} \frac{w(s)}{w(r)}-1\right] \mathrm{d} r=0
$$




$$
\begin{gathered}
\int_{-1}^{1}\left(1-r^{2}\right)^{-1 / 2} \phi(r) \mathrm{d} r=1 \\
\int_{-1}^{1} \frac{\left(1-r^{2}\right)^{-1 / 2} \phi(r)}{w(r)} \mathrm{d} r=\frac{P}{\beta Q} \\
p(s)=\frac{\beta Q}{\pi L} w(s) \int_{-1}^{1} \frac{\left(1-r^{2}\right)^{-1 / 2} \phi(r)}{w(r)(r-s)} \mathrm{d} r .
\end{gathered}
$$

The system of eqns (20)-(23) is discretized by applying the method of Erdogan and Gupta [12]. Equations (20) and (21) yield a system of $n$ algebraic equations for the $n$ discretized values of $\phi(r)$. Equation (22) and the requirement of vanishing normal stress of $-a$, or

$$
\int_{-1}^{1} \frac{\left(1-r^{2}\right)^{-1 / 2} \phi(r)}{w(r)\left(r+\frac{a}{L}\right)} \mathrm{d} r=0
$$

suffice to determine $a / L$ and $b / L$ by iteration. It was found computationally that, for $\beta$ positive, $a / L$ is extremely close to 1 , or that the extent of the left contact zone is of the order of $10{ }^{8} \mathrm{~L}$ or smaller. Although a/ $L$ could not be determined exactly, it did not affect the numerical results because of this fact. Similarly, (24) could not be used. Moreover, to avoid iterations, $b / L$ was specified and the required ratio $P / Q$ was computed from (22). The normal stress over the bond and the right contact zone was computed from (23). Recalling the relation between the shear and normal stress intensity factors at $\pm L[1,2]$

$$
K_{\mathrm{I}}\left( \pm L^{+}\right)=\mp \beta K_{2}( \pm L)
$$

it is sufficient to show that $K_{2}(-L)$ is negative for the contact stress on the left zone to be compressive. This indeed was the case. In addition, the integral in (23) changed sign rapidly indicating that a value of $a / L$ extremely close to 1 can be found so that (24) is satisfied. It should be noted that if $\beta$ is taken as negative, the two zones are interchanged. Finally, the slope of the gap is obtained as

$$
\frac{\mathrm{d} g}{\mathrm{~d} x}(s)=\frac{\beta Q}{4 \pi L}\left[\frac{\kappa_{1}+1}{\mu_{1}}+\frac{\kappa_{2}+1}{\mu_{2}}\right]\left|\frac{s-\frac{b}{L}}{s+\frac{a}{L}}\right| 1 / 2 \int_{-1}^{1} \frac{\left(1-r^{2}\right)^{-1 / 2} \phi(r)}{w(r)(s-r)} \mathrm{d} r, \quad|s|>1
$$

and it was verified that the gap remained positive over the separation zones. Figure 2 shows

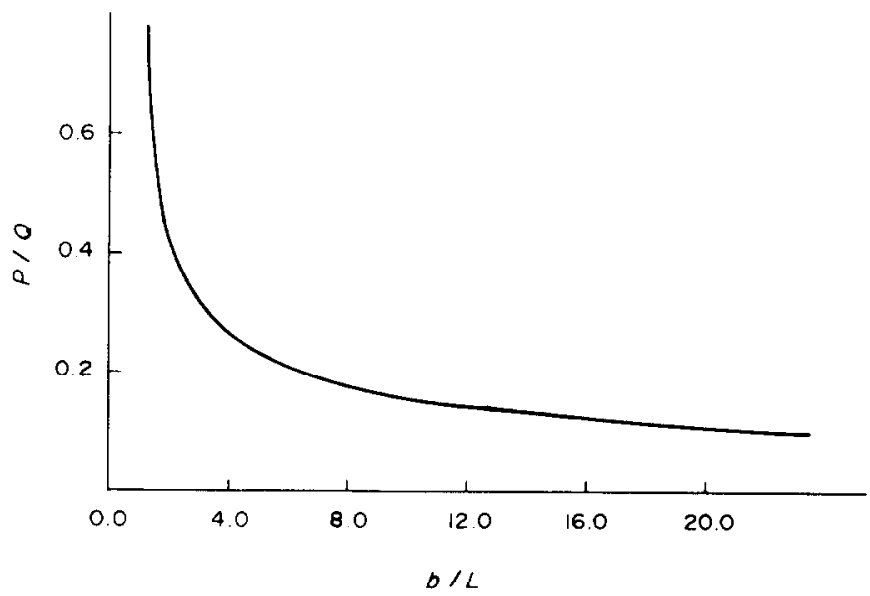

Fig. 2. Variation of $P / Q$ with $b / L(\beta=0.5)$. 


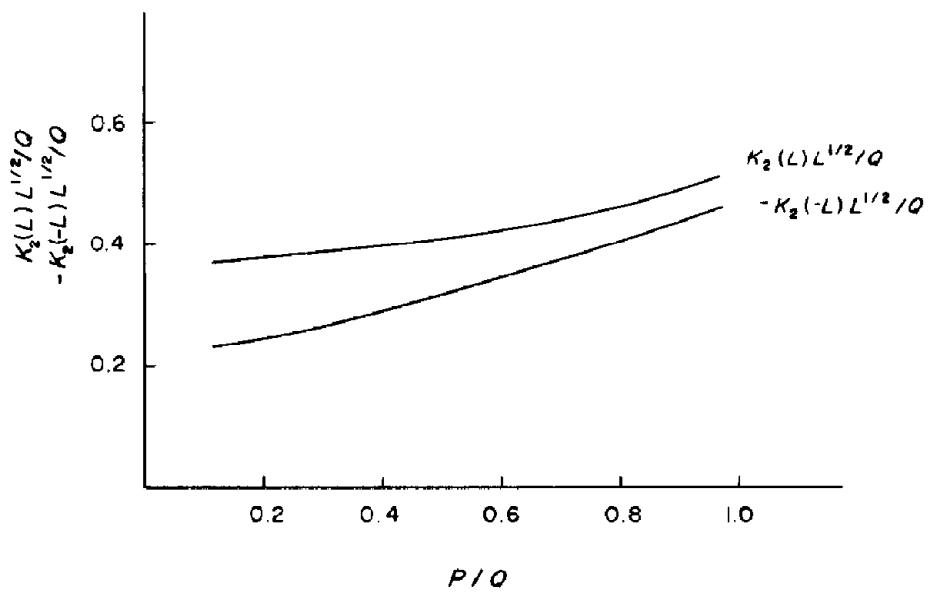

Fig. 3. Variation shear stress intensity factors with $P / Q(\beta=0.5)$.

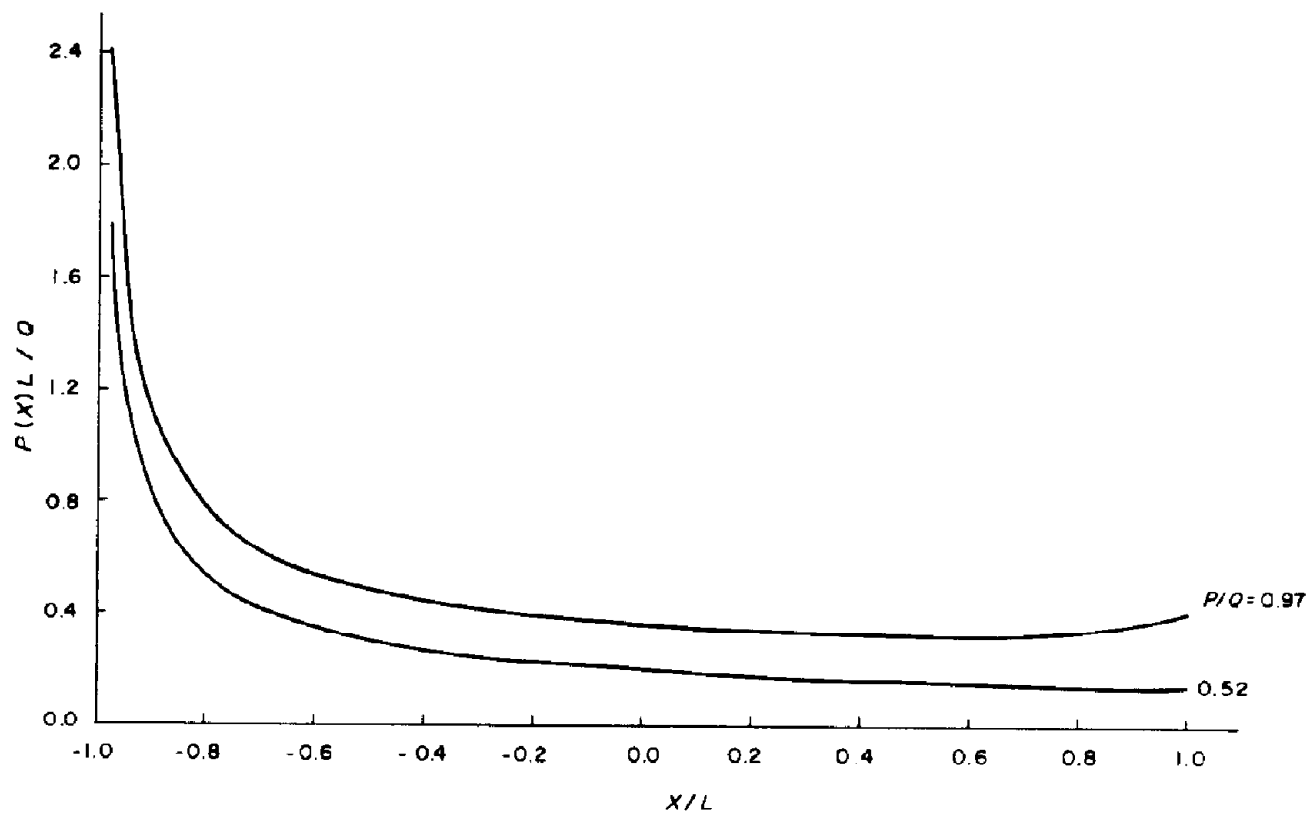

Fig. 4. Normal stress over the bonded zone $(\beta=0.5)$.

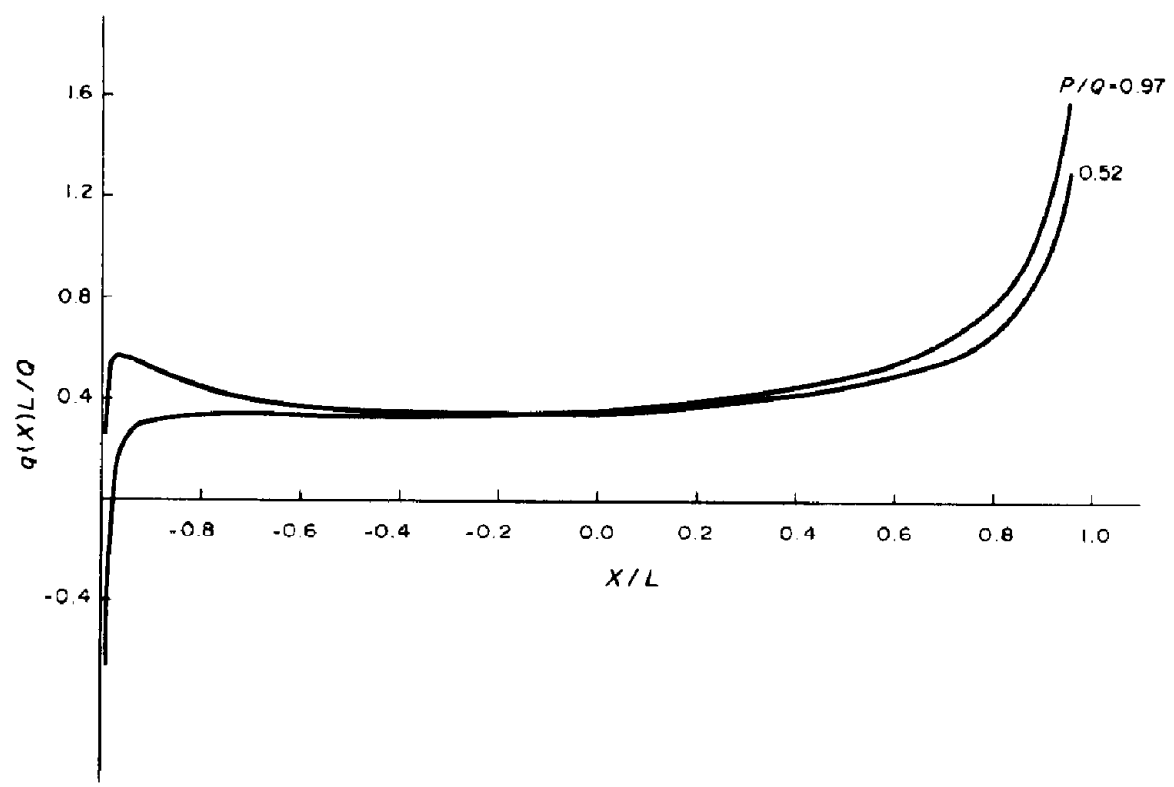

Fig. 5. Shear stress over the bonded zone $(\beta=0.5)$. 


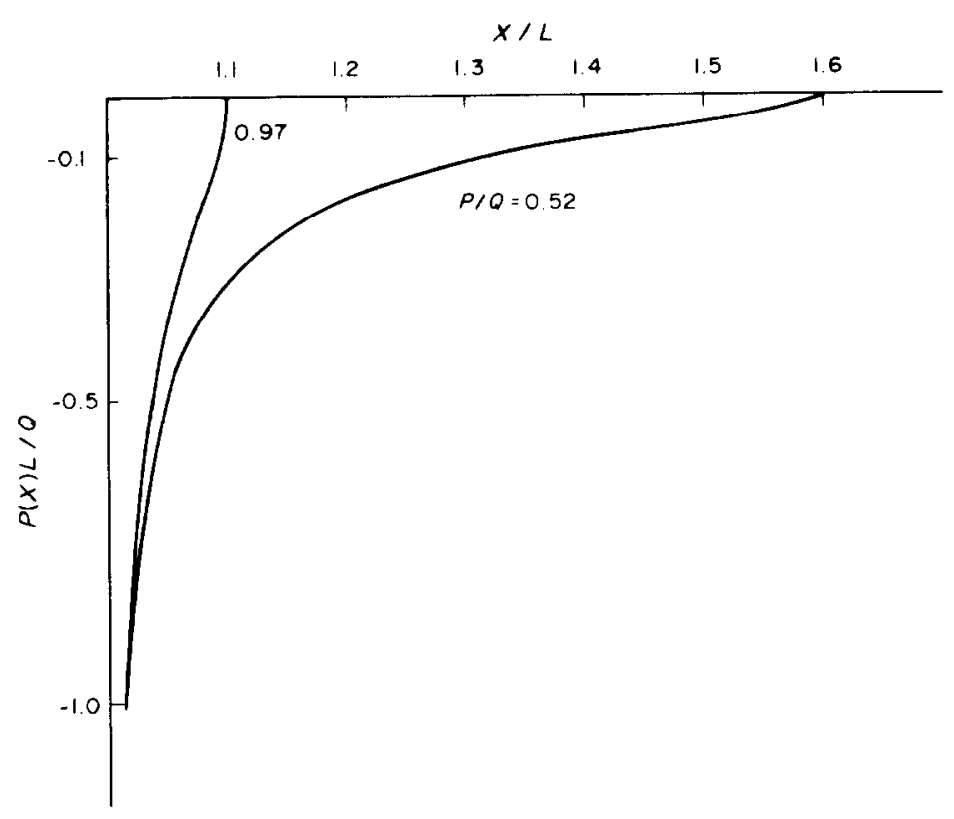

Fig. 6. Normal stress over the right contact zone $(\beta=0.5)$.

how $b / L$ varies with $P / Q$. As $P / Q \rightarrow 0, b / L$ approaches asymptotically $x$, viz. under the action of shear only, the right edge crack closes completely. The left edge crack will start closing when compression is applied, but it can never close completely [4]. The variation of the shear stress intensity factors with $P / Q$, is given in Fig. 3. Although the results for $K_{2}(L)$ are accurate, $K_{2}(-L)$ is accurate only to the first decimal point, and this accuracy is obtained by using 200 collocation points (a bigger matrix could not be handled numerically). The normal stress over the bond is shown in Fig. 4 for two values of the ratio $P / Q=0.97,0.52$ which correspond to $b / L=1.1,1.6$. The values at $\pm L$ are both finite, although at $-L p(x)$ is very large. Figure 5 gives the shear stress over the bond for the same values of $P / Q$, and Fig. 6 shows the normal stress over the right contact zone. In all results shown $\beta$ was taken as 0.5 .

Acknowledgement - The author would like to acknowledge the support by the National Science Foundation through the grant ENG $77-25032$ during the course of this research.

\section{REFERENCES}

[1] M. COMNINOU, J. Appl. Mech. 44, 631 (1977).

[2] M. COMNINOU, J. Appl. Mech. 45, 287 (1978).

[3] A. H. ENGLAND, J. Appl. Mech. 32, 400 (1965).

[4] M. COMNINOU and D. SCHMUESER, J. Appl. Mech. 46, 345 (1979).

[5] L. M. KEER, S. H. CHEN and M. COMNINOU, Int. J. Engng Sci. 16, 765 (1978).

[6] D. SCHMUESER and M. COMNINOU, Int. J. Solids Structures 15, 927 (1979).

[7] W. JANACH, Int. J. Fract. 14, R325 (1978).

[8] F. ERDOGAN, J. Appl. Mech. 30, 232 (1965).

[9] S. P. TIMOSHENKO and J. N. GOODIER, Theory of Elasticity. McGraw-Hill, New York (1970).

[10] I. STAKGOLD, Boundary Value Problems of Mathematical Physics, Vol. II. Macmillan, London (1968).

[11] N. I. MUSKHELISHVILI, Singular Integral Equations. Noordhoff, Leyden (1953).

[12] F. ERDOGAN and G. GUPTA, Q. App. Math. 29, 525 (1972). 